\title{
Cellular expression of lymphocyte function associated antigens and the intercellular adhesion molecule-1 in normal tissue
}

\author{
ME F Smith, J A Thomas
}

\begin{abstract}
A detailed immunohistological analysis of normal tissues for the distribution of lymphocyte function-associated antigens (LFA) and the intercellular adhesion molecule-1 (ICAM-1) showed several hitherto unrecognised patterns of LFA-3 and ICAM-1 expression. The widespread, but not ubiquitous, distribution of LFA-3 contrasted with the more restricted distribution of ICAM-1. Among epithelial cells, all tissues which were ICAM-1 positive were also LFA-3 positive with the single exception that thymic cortical epithelium, in contrast to previous reports, expressed only ICAM-1. It was striking that LFA-3 molecules were absent in some tissues which are considered to be sites of immunological privilege (such as brain and testis), suggesting an additional mechanism by which these microenvironments maintain immunological autonomy. Furthermore, the unexpected finding that LFA-3 is strongly expressed on intercalated discs of cardiac muscle may possibly be related to a nonimmune function, or indicate a structurally similar epitope expressed by an unrelated molecule within this tissue.
\end{abstract}

Leucocyte adhesion mediated by non-antigen specific receptor/ligand interactions is important in many immune responses involving $T$ cell recognition of specific antigen as well as other leucocyte effector functions. The structural and functional characterisation of a group of lymphocyte surface molecules mediating these interactions has emerged largely through the production of specific monoclonal antibodies which directly inhibit conjugate formation between effector lymphocytes and their corresponding target cells.

LFA-1 is exclusively expressed on haemopoietic cells and belongs to a family of heterodimeric adhesion proteins known as integrins. ${ }^{12}$ ICAM-1 has been identified as a ligand for LFA-1 and is expressed on leucocytes and various non-haemopoietic cells. $^{34}$ Inhibition studies with monoclonal antibodies to LFA-1 and ICAM-1 indicate that binding between this receptor-ligand pair is an important initial event in stabilising intercellular contacts for antigen dependent $T$ cell proliferation, ${ }^{5}$ lytic functions of cytotoxic $T$ cells (CTL), ${ }^{126}$ and natural killer cells, ${ }^{2}$ and in $T$ dependent $B$ cell activation ${ }^{7}$ in vitro.
Increases in ICAM-1 expression on dermal fibroblasts, ${ }^{34}$ endothelial cells, ${ }^{48}$ and keratinocytes ${ }^{910}$ in culture induced by cytokines are closely associated with increased adhesion to these cells by activated $\mathrm{T}$ lymphocytes and other leucocytes. These observations suggest that LFA-1/ICAM-1 dependent cytoadhesion is also an important mechanism whereby leucocyte circulation and inflammatory responses can be regulated in vivo. There are also strong indications based mainly on amino acid sequence analysis that ICAM-1 is the major human rhinovirus receptor. ${ }^{11}$ A separate but interrelated pathway controlling nonantigen specific $T$ cell adhesion is mediated by LFA-3 which acts as the ligand for the CD2 (also known as LFA-2) T cell specific surface receptor. ${ }^{12}$ LFA-3 is widely distributed on peripheral blood leucocytes, thymocyte subpopulations, and non-haemopoietic cells of different histogenic origins. ${ }^{12}$ Interactions regulated by LFA-3/CD2 are involved in forming antigen independent cellular conjugates required for $T$ cell activation and proliferation in response to antigen, ${ }^{12}$ cooperative functions with B cells, ${ }^{13}$ and CTL activity. ${ }^{12}$ LFA-3 is also thought to participate actively in intercellular signalling and signal transduction. Thus LFA-3/CD2 binding may influence intrathymic $\mathrm{T}$ cell proliferation. Furthermore, the secretion of $T$ cell activating cytokine IL1 by thymic epithelial cells can be induced with monoclonal antibodies to LFA-3 in vitro. $^{14}$

The extent to which LFA molecules maintain the integrity of the immune system is highlighted by their changed expression in certain pathological conditions. Epidermotropic $T$ cell infiltrates closely correlate with de novo ICAM-1 expression on keratinocytes in inflammatory skin diseases. ${ }^{10}$ Conversely, reduced or absent expression of LFA and ICAM-1 molecules on high grade nonHodgkin's lymphoma, ${ }^{15}$ on Burkitt's lymphoma, and on early derived Burkitt's lymphoma cell lines in culture ${ }^{16}$ and colorectal adenocarcinoma ${ }^{17}$ suggests that loss of these molecules contributes to tumour progression by allowing the malignant cells to escape immunosurveillance. Previous studies have documented the distribution of LFA-1, ${ }^{12}$ CD2 (LFA-2), ${ }^{12}$ ICAM-1 ${ }^{4}$ and, to a lesser extent, LFA $-3^{212}$ molecules in selected normal tissues. This immunocytochemical study was carried out using an extensive panel of tissues to compare the normal phenotypic expressions of LFA and ICAM-1, particularly with respect to previously uninvestigated body 
sites. Given the importance of these molecules in immune regulation, the aim of this investigation was not only to elucidate hitherto unrecognised sites of expression but also to provide a comprehensive guide on which to interpret patterns of change occurring in different disease states.

\section{Methods}

Immunocytochemical studies were performed on human lymphoid, epithelial, and mesenchymal tissues. Thirty nine tissue samples were obtained from many different body sites, either as surgical biopsy or resection samples, or as necropsy samples within 24 hours of death. The type and number of tissues obtained from different cases were as follows: neonatal/child thymus $(\mathrm{n}=5)$; tonsil $(\mathrm{n}=3)$; lymph node $(\mathrm{n}=1)$; spleen $(\mathrm{n}=1)$; oral mucosa $(\mathrm{n}=1)$; oesophagus $(n=1)$; stomach $(n=1)$; colon $(n=5)$; liver $(n=2)$; lung $(n=2)$; bronchus $(\mathrm{n}=1)$; heart $(\mathrm{n}=1)$; kidney $(\mathrm{n}=1)$; urinary bladder $(n=1)$; thyroid gland $(n=1)$; adrenal gland $(n=1)$; salivary gland $(n=2)$; skin $(n=1)$; brain $(n=1)$; testis $(n=1)$; fallopian tube $(n=1)$; ectocervix $(n=1)$; placenta $(n=4)$. With the exception of tonsil tissue, which showed reactive hyperplasia, all samples were macroscopically and microscopically normal. Samples were snap frozen in isopentane cooled in liquid nitrogen, and stored under liquid nitrogen, or at $-70^{\circ} \mathrm{C}$, before cryostat sectioning at $7 \mu \mathrm{m}$ thickness.

Tissue section analysis was performed on chloroform-acetone fixed cryostat sections using the peroxidase-antiperoxidase (PAP) method. ${ }^{18}$ Specific receptor determinants were identified with monoclonal antibody T11 (Coulterclone) directed against the CD2 T cell receptor; TS2/9, which defines the 60 kilodalton CD 58 antigen, LFA- $3^{2}$; RR1/1, specific for the 90-114 kilodalton form of the CD 54 antigen, ICAM- $1^{3}$; and monoclonal antibody TS $1 / 22$ which is directed against the 177 kilodalton $\alpha$ chain subunit (CD11a) of LFA-1. ${ }^{2}$ The monoclonal antibodies to LFA-1, LFA-3, and ICAM-1 were kindly provided by $\operatorname{Dr} T$ A Springer, Center for Blood Research, Harvard Medical School, Massachusetts, USA. Additional monoclonal antibodies specific for HLA-DR $\propto$ (Tal 1B5; obtained through the Tissue Antigen Laboratory, Imperial Cancer Research Fund, London and CDla (Nal/34;

Cellular expression of $L F A-3$ and ICAM-1 in normal human tissues

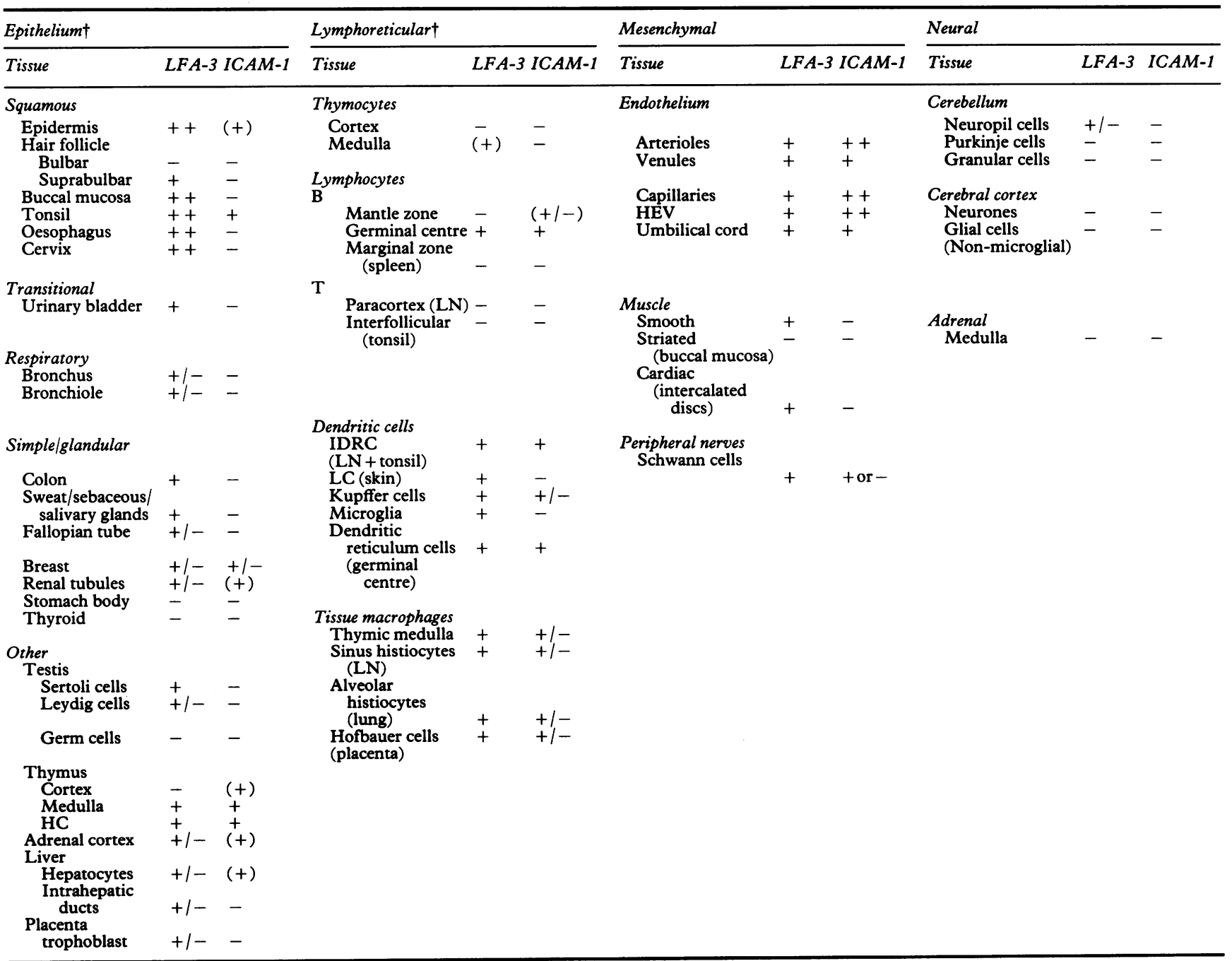

* Staining intensity reported as $++=$ intense; $+=$ strong; $+1-=$ weak; $-=$ absent; $(+)=$ staining of minority cell populations. $\dagger$ HC: Hassall's corpuscle: ¿ Staining intensity reported as $++=$ intense; $+=$ strong; + interdigitating reticulum cell; LC: Langerhans' cell; HEV: high endothelial venule. 
Figure 1 Buccal mucosa: intense $L F A-3$ positive staining of stratified squamous epithelium.

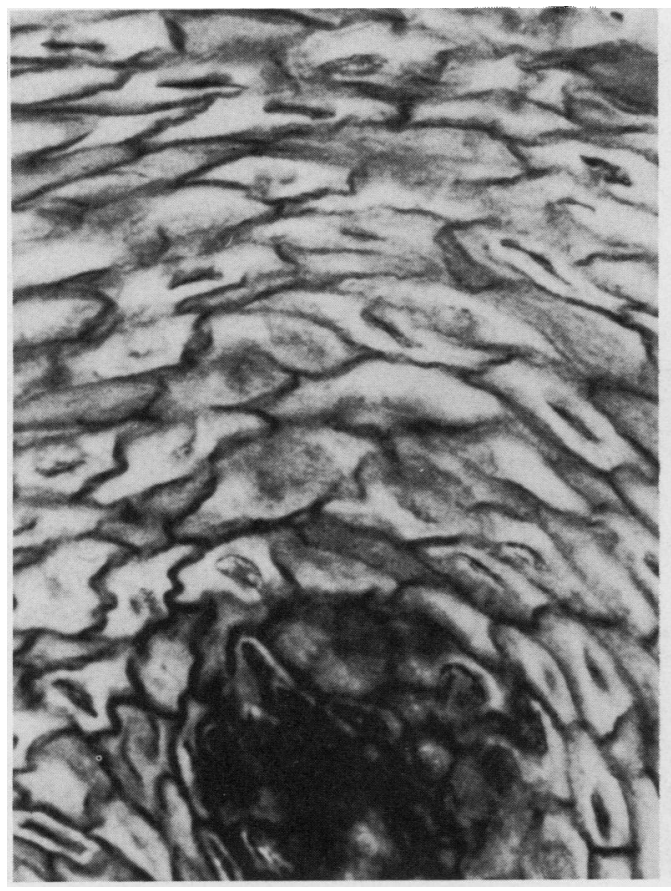

Seralab) were used to identify specialised antigen presenting cells of dendritic morphology in certain tissues. Supernatant or ascites preparations were used at saturating dilutions. Modifications of the standard PAP technique used to minimise non-specific tissue reactivity included pre-incubation of the sections with normal rabbit serum (1/10) for 20 minutes before the application of the primary antibody. Antibody labelling was performed with rabbit anti-mouse immunoglobulin (Dakopatts; Z259) containing normal human serum both at a 1 in 25 final dilution, and mouse PAP (Dakopatts, P850) at a 1 in 100 dilution. All antibody dilutions were made in phosphate buffered saline (PBS). All antibody incubations were performed at room temperature in a damp chamber for $45-60$ minutes with

Figure 2 Testis: cells close to the seminiferous tubule boundary $(\leftarrow)$ reminiscent of Sertoli cells, show intense cytoplasmic staining for $L F A-3$.

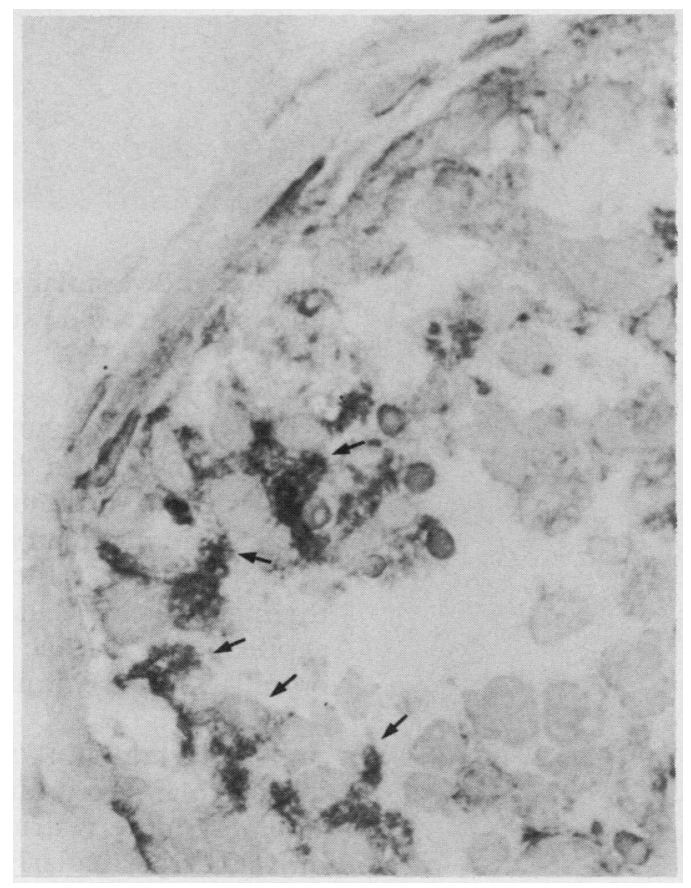

intervening rinses in PBS. The peroxidase label was developed with 3,3'-diaminobenzidine tetra-hydrochloride (Sigma; D05637); sections were counterstained in Mayer's haemalum before dehydration and permanent mounting.

\section{Results}

The reactivity patterns of LFA-3 and ICAM-1 in various tissues are shown in the table.

\section{TISSUE DISTRIBUTION OF LFA-3}

Cell types which consistently expressed abundant amounts of LFA-3 included tissue macrophages in lymph node (sinus histiocytes), lung (alveolar macrophages), and placenta (Hofbauer cells) as well as all blood vessels, including specialised high endothelial venules in peripheral lymphoid tissues.

In primary and secondary lymphoid tissues positive staining was largely restricted to nonlymphoid components within the thymus, which included medullary epithelial cells, Hassall's corpuscles, and macrophages, although membrane staining on a small proportion of mononuclear cells consistent with medullary thymocytes was observed in all samples. No staining was observed on cortical thymocytes, or subcapsular, or cortical epithelial cells. Whereas most lymphocytes in the mantle zone $B$ cell areas, $T$ cell areas in the paracortex, and interfollicular compartments of peripheral lymph node and reactive tonsils were negative for LFA-3, all germinal centre cells within secondary B cell follicles exhibited strong LFA-3 activity. In contrast, no lymphoid staining was observed in spleen.

Epithelial tissues in different body sites showed considerable variation in LFA-3 expression. Strong membrane staining was observed on stratified squamous epithelium in skin (epidermis), buccal mucosa (fig 1), cervix, and oesophagus, on transitional cell epithelium of urinary bladder and on colonic mucosal epithelium. Although dermal appendages in skin (sweat glands and sebaceous glands) were also strongly LFA-3 positive, hair follicle epithelium showed a distinct pattern with strong staining on suprabulbar portions, contrasting with the lack of staining on bulb epithelium surrounding the dermal papillae. Heterogeneous LFA-3 staining was also noted in the testis. The extensive cytoplasm of Sertoli cells exhibited strong LFA-3 activity (fig 2); germ cells were not stained. Numerous epithelia showed moderate or weak expression of LFA-3. Moderate LFA-3 positive staining was observed on renal tubular cells (fig 3 ); weak staining was usual on breast acini and ducts, hepatocytes, bile ducts within portal tracts, fallopian tube, adrenal cortex, bronchus and terminal bronchiole. No LFA-3, however, was detected on the epithelium of gastric body and thyroid follicular epithelium.

The only cells to show strong positive staining in neural tissues were microglial cells of the cerebral and cerebellar cortex (fig 4A), although weak diffuse activity was present on the neuropil molecular layer of cerebellum. No 
Figure 3 Kidney: diffuse $L F A-3$ positive staining of proximal tubule $(p)$ epithelial cells. Distal tubules (d) lined by epithelial cells with central, spherical nuclei are LFA-3 negative ( $g=$ glomerulus)

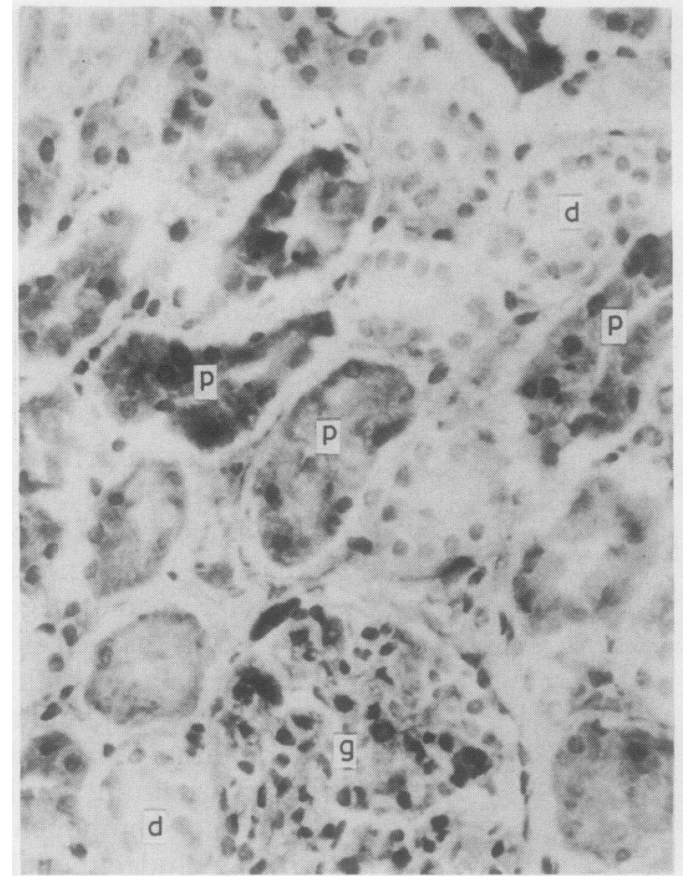

staining was seen on cerebral cortical neurones, glial cells (other than microglia), cerebellar Purkinje and granular cells, or on cells of the adrenal medulla.

Highly dendritic cells, which were mor- phologically consistent with specialised antigen presenting cells, including interdigitating reticulin cells (IDRC) in peripheral lymphoid tissue, hepatic Kupffer cells (fig 4B), and cerebral microglia (fig 4A), expressed abundant amounts of LFA-3. Despite the network of intense membrane staining on epidermal keratinocytes and other stratified squamous epithelia, close microscopic examination showed a population of LFA-3 positive intraepithelial dendritic cells similar to Langerhans' cells in some of these tissues (fig 4C). To confirm this observation, consecutive sections of buccal mucosa and skin were examined for HLA-DR and CD1a antigens which are known to be expressed on most antigen presenting cells in different body sites. Only a small proportion of intraepithelial dendritic cells expressing HLA-DR and CDla antigens also expressed LFA-3.

Mesenchymal cells, Schwann cells of peripheral nerve, vascular and visceral smooth muscle cells and blood vessel endothelial cells, which were identified in many tissues, were consistently LFA-3 positive. Strong staining was also detected on intercalated discs of cardiac muscle (fig 5); skeletal muscle, however, was LFA-3 negative.

Placental tissue at full term showed weak positive staining of villous trophoblasts. In the umbilical cord LFA-3 was present on endo-
Figure 4 (A)

Cerebellum: $L F A-3$ positive staining of cortical microglia.

(B) Liver: LFA-3 positive staining of Kupffer cells in hepatic sinusoids. (C) Skin: LFA-3 positive staining on epidermal dendritic cell morphologically reminiscent of Langerhans' cells.

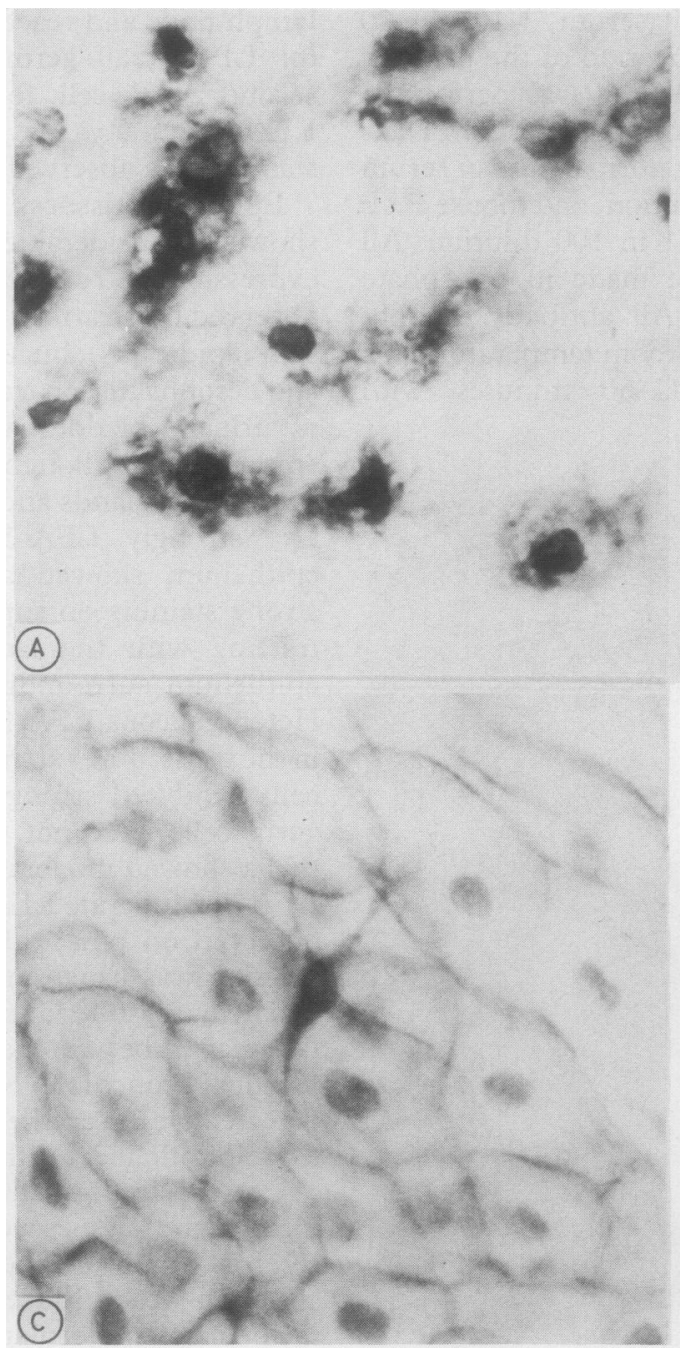

thelium and vascular smooth muscle; no staining was observed on stromal cells of Wharton's jelly.

CD2 ANTIGEN DISTRIBUTION IN NORMAL TISSUE The expression and distribution of CD2 on T cells is well documented. In thymus, both cortical and medullary lymphocytes were CD2 positive whereas there was no staining of thymic epithelial cells. CD2 antigens were not expressed on cardiac muscle components nor on cells within seminiferous tubules of testis.

TISSUE DISTRIBUTION OF ICAM-1

The cellular distribution of ICAM-1 was considerably more restricted compared with that of LFA-3, particularly on epithelium (table). 


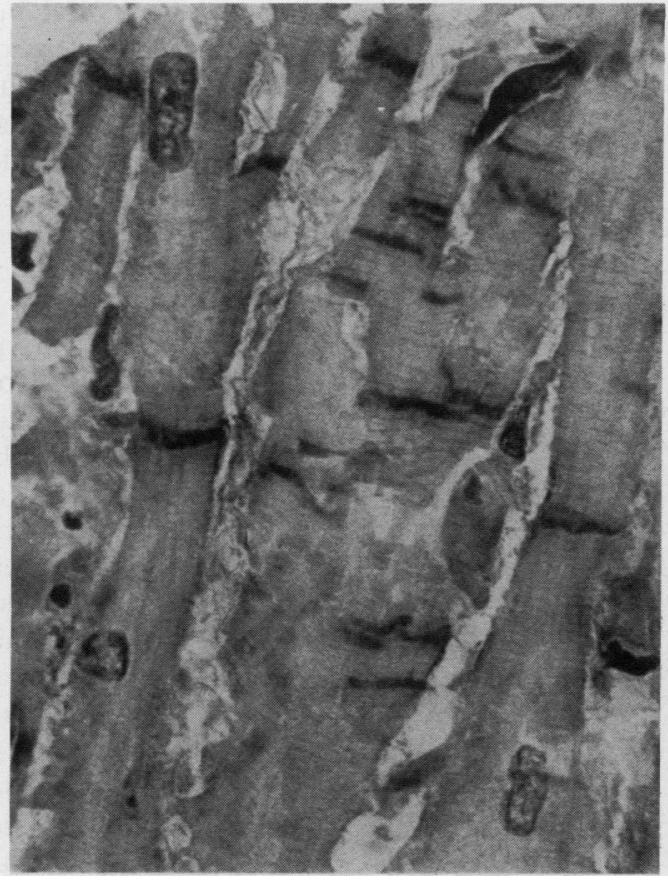

Figure 5 Cardiac muscle: intense LFA-3 positive staining of intercalated discs and on capillary endothelium between muscle fibres.

In lymphoid tissues ICAM-1 was present on thymic medullary epithelium, Hassall's corpuscles, and on a few cortical epithelial cells. Some positive cells in the cortex were morphologically similar to thymic nurse cells (fig 6) which have been reported to have important influences on intracortical thymocyte differentiation. ${ }^{19}$ Outer cortical and subcapsular epithelium, as well as medullary and cortical thymocytes, were ICAM-1 negative. In tonsil staining was present, albeit weakly, on a few

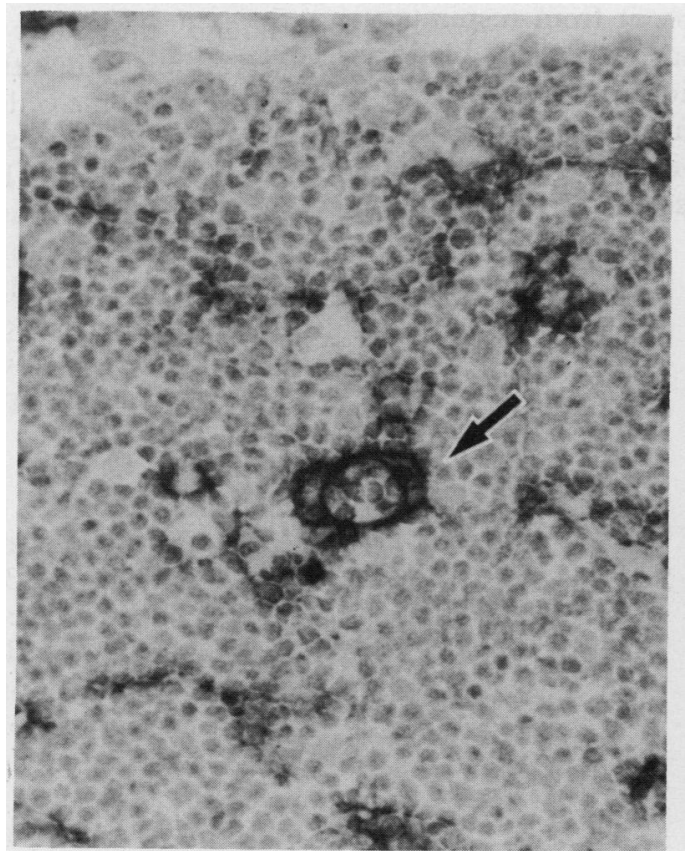

Figure 6 Thymus:ICAM-1 positive staining on low numbers of cortical epithelial cells. A circular arrangement of ICAM-1 positive epithelial cells surrounding a focus of cortical thymocytes resembles a thymic nurse cell (arrow). Outer cortical epithelium and cortical thymocytes are ICAM-1 negative.

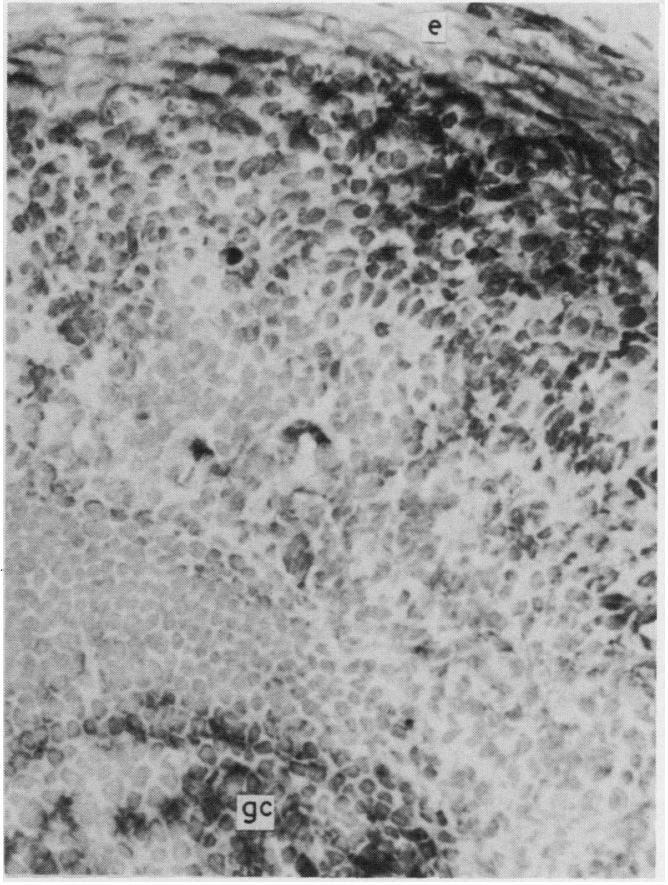

Figure 7 Tonsil: ICAM-1 positive staining on stratified squamous epithelium (e) and germinal ( $g c$ ) centre cells.

mantle zone B lymphocytes. Germinal centre cells, however, showed two different patterns of expression. In one type, which was rarely seen, positive staining showed an intense follicular dendritic pattern with intervening areas of ICAM-1 negative cells. A more common finding was for all germinal centre cells to stain for ICAM-1 without distinguishing the dendritic reticulum cell network. T cell areas in lymph node and tonsil were ICAM-1 negative.

Among reticuloendothelial cells, sinus his-

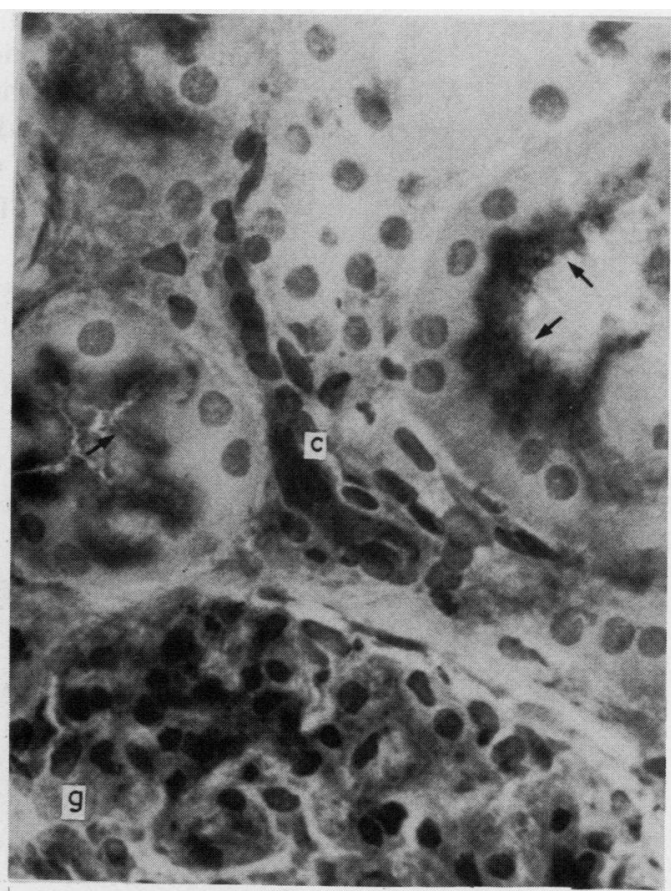

Figure 8 Kidney: strong ICAM-1 positive staining of the luminal surface of proximal renal tubule cells $(\rightarrow)$. Interstitial capillaries $(\mathrm{c})$ and glomerulus $(\mathrm{g})$ are also ICAM positive. 


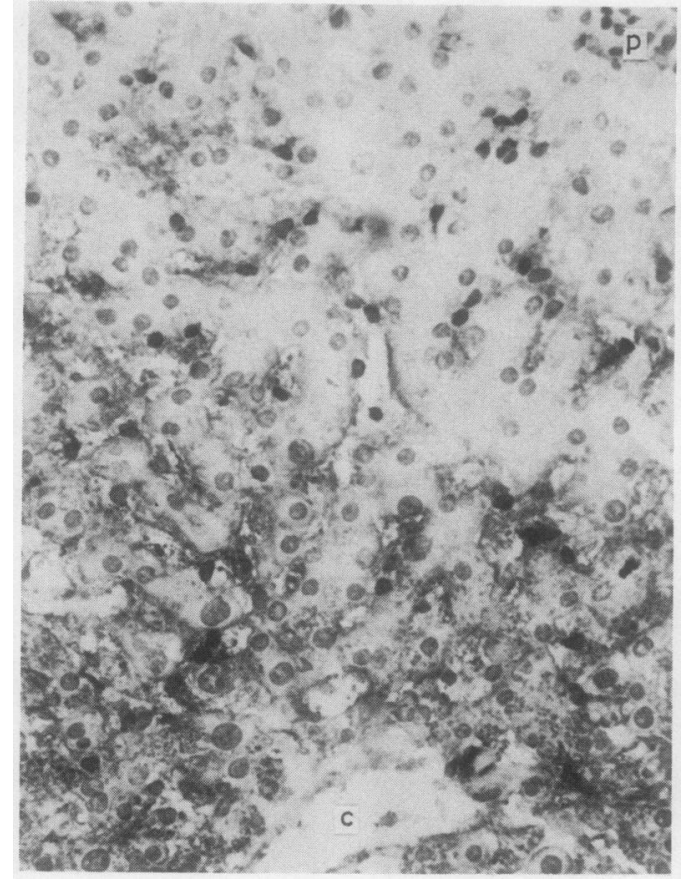

Figure 9 Liver: localised ICAM-1 positive staining on hepatocytes in centrilobular areas round central vein $(c)$ contrasts with the absence of staining in periportal $(p)$ cells.

tiocytes, placental Hofbauer cells and pulmonary alveolar macrophages showed weak activity.

In epithelial tissues only stratified squamous epithelia in tonsil showed strong staining for ICAM-1 (fig 7). Staining was absent on oesophageal, cervical, and buccal mucosal epithelium, and in the epidermis, apart from a focus of ICAM-1 positive staining related to a small lymphocyte infiltrate. Focal staining was also present at the luminal edge of epithelial cells of proximal convoluted tubules in kidney (fig 8). ICAM-1 was absent from respiratory epithelium of bronchus, urinary bladder transitional cell epithelium, gastric body and colonic epithelia, and from Leydig cells and seminiferous tubule epithelium of testis. In liver, ICAM-1 was present on centrilobular

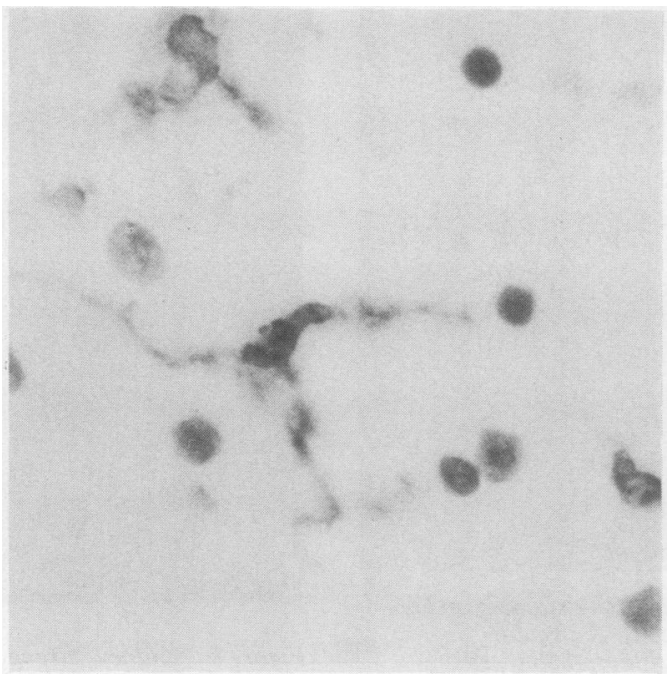

Figure 10 Cerebellum: LFA-1 positive staining of microglial cell. but not periportal hepatocytes (fig 9). Weak staining was observed in the deep portions of adrenal cortex (zona reticularis) but not in more superficial areas constituting the zona glomerulosa and fasciculata.

In neural tissues expression was limited to capillary endothelium. In placental tissue villous trophoblast was ICAM-1 negative. Dendritic cells with similar morphology and location as antigen presenting cells showed variable ICAM-1 expression at different body sites. Whereas no staining of typical Langerhans' like cells was observed in skin and other stratified squamous epithelia, or on cerebral microglia, large numbers of cells similar to Kupffer cells and interdigitating reticulim cells in liver and lymphoid tissue, respectively, showed positive staining.

ICAM-1 stained arteriolar, capillary, and high endothelial venule endothelium intensely, but staining of non-specialised venules was highly variable and occasionally absent. Similar variability was observed in Schwann cells. All types of muscle cells were ICAM-1 negative.

TISSUE DISTRIBUTION OF LFA-1

LFA-1 expression was confined to tissues of haemopoietic origin. In thymus positive staining was seen on medullary and cortical thymocytes but not on epithelial or endothelial cells. Small lymphocytes in tonsil mantle zones were weakly positive in contrast to $T$ (paracortical and interfollicular zone) lymphocytes and germinal centre cells which were uniformly positive. Dendritic cells, morphologically reminiscent of antigen presenting cells in some tissues showed different patterns of LFA-1 expression when compared with that of LFA3 , suggesting variations in the cellular distribution of the epitopes recognised by the anti-LFA monoclonal antibodies used in this study. Although the same intense staining was observed for both antigens, the long dendritic processes of some cells, cerebral microglia (fig 10 ), and Kupffer cells in particular, were well delineated with anti-LFA-1; whereas shorter and more numerous fine processes were demonstrated with anti-LFA-3 (figs 4A, B). It was surprising to find, however, that no staining of Langerhans'-like cells was observed with the anti-LFA-1 monoclonal antibody in stratified squamous epithelia.

\section{Discussion}

Several of the observations on the normal distribution of LFA-3 and ICAM-1 described in this study have not been previously documented. The findings for LFA-1 and CD2 corroborate those of previous reports in that no new sites of expression were shown. Although LFA-3 expression was widespread, it was not present in all tissues. The presence of LFA-3 on dendritic cells that were morphologically similar to epidermal Langerhans' cells, hepatic Kupffer cells, and cerebral microglia is consistent with the specialised function of these cells in presenting antigen to effector $T$ cells. The presence of an LFA-3/CD2 dependent 
adhesion pathway would make an important contribution to strengthening the interaction between cells presenting antigen and effector $T$ lymphocytes. The lack of detectable LFA-3 on germ cells of seminiferous tubules is reminiscent of the reported lack of HLA-ABC major histocompatibility antigens on these cells. ${ }^{20} \mathrm{It}$ may be that the failure to express LFA-3 also contributes to a protective mechanism by which mature spermatozoa can escape maternal CTL responses directed against foreign paternal antigens. Similarly, the lack of LFA-3 staining on most cells in cerebral tissue, as well as in testis, may also be important for maintaining the immunological privilege associated with these sites. The failure to detect LFA-3 on thymic cortical epithelium in this study contrasts with a previous report in which LFA-3 was shown throughout the thymic reticular network. ${ }^{12}$ In vitro studies suggest that intrathymic $\mathrm{T}$ cell activation is mediated through binding between $\mathrm{CD} 2$ receptor on the thymocyte surface and LFA-3 expressed on thymic epithelium, ${ }^{21}$ particularly in the thymic subcapsular cortex. ${ }^{22}$ From our observations, however, it seems unlikely that cortical epithelial cells are involved in these interactions in vivo.

ICAM-1 showed a limited range of tissue expression. Most epithelial cells were ICAM-1 negative, although the focus of positive ICAM1 expression in skin at the site of minimal $\mathrm{T}$ cell infiltration reflects both the pronounced increases in ICAM-1 expression exhibited by cultured keratinocytes in response to lymphokine stimulation, ${ }^{9}$ and recent observations that in inflammatory skin conditions the induction of ICAM-1 on epidermal keratinocytes closely correlates with the presence of epidermotropic T cell populations. ${ }^{10}$

Recent protein sequence analyses have implicated ICAM-1 as the major binding receptor for most of the rhinoviruses which cause the common cold. ${ }^{11}$ Of all normal tissues available for study, present findings that ICAM-1 positive staining of normal stratified squamous epithelium is restricted to the tonsil may be important as the oropharyngeal epithelium, forming the tonsilar surface and crypts, is a common site of exposure to rhinovirus infection. The focal expression of ICAM1 on adrenal cortical cells observed in this study has not been reported previously. Our observations of focal ICAM-1 positive staining on centrilobular hepatocytes, however, disagrees with the findings of two reports in which predominantly sinusoidal staining for ICAM-1 was observed in normal necropsy ${ }^{4}$ and nontransplanted donor liver samples. ${ }^{23}$ Similarly, our findings of ICAM-1 expression on proximal tubules of normal kidney fails to support the absence of normal renal tissue staining in other reports. ${ }^{44}$ Adams et al have recently suggested that the increased expression of ICAM-1 localised to centrilobular hepatocytes during acute rejection of liver allografts may be an important factor in the development of inflammatory responses during graft rejection. ${ }^{23}$ Similarity between the distribution of ICAM-1 reported in liver allografts during acute rejection and the present findings in histologically normal liver suggest that other mechanism(s) might induce focal centrilobular patterns of ICAM-1 in normal hepatic tissue. Faul and Russ have suggested that increases in ICAM-1 expression in transplanted renal tissue could also result from ischaemic damage. ${ }^{24}$ The inevitable hypoxic changes that take place in necropsy tissue may influence variations in ICAM-1 expression as the centrilobular areas, which showed the most intense staining for ICAM-1 in normal liver, are the sites most distant from the arterial blood supply. On the other hand, the restricted ICAM-1 expression observed in many tissues may indicate the presence of additional LFA-1 ligands. In this context the cDNA of an alternative LFA-1 ligand, ICAM2 , has recently been cloned. ${ }^{25}$ The tissue distribution of this molecule, which is smaller than ICAM-1, has not been fully investigated. Nevertheless, there is clear evidence that the expression of ICAM-1 on specific cell types is regulated by cytokine activity in vitro and in vivo and can be changed or rapidly induced in certain pathological conditions. Whether the constitutive absence of ICAM-1 in many normal tissues identified in this study is also subject to modulation by similar mechanisms remains to be elucidated.

1 Springer TA, Dustin ML, Kishimoto TK, Marlin SD. The lymphocyte function-associated LFA-1 CD2, and LFA-3 molecules: cell adhesion receptors of the immune system. Ann Rev Immunol 1987;5:223-52.

2 Krensky AM, Sanchez-Madrid F, Robbins E, Nagy JA, Springer TA, Burakoff SJ. Functional significance, distribution, and structure of LFA-1, LFA-2 and LFA-3: cell surface antigens associated with CTL-target interaccell surface antigens associated with

3 Rothlein R, Dustin ML, Marlin SD, Springer TA. A human intercellular adhesion molecule (ICAM-1) distinct from intercellular adhesion molecule (ICAM-1. J Immunol 1986;137:1270-4.

4 Dustin ML, Rothlein R, Bhan AK, Dinarello CA, Springer $\mathrm{TA}$. Induction by $\mathrm{IL} 1$ and interferon- $\gamma$ : tissue distribution, biochemistry, and function of a natural adherence molecule (ICAM-1). J Immunol 1986;137:245-54.

5 Shaw S, Ginther Luce GE. The lymphocyte functionassociated antigen (LFA)-1 and CD2/LFA-3 pathways of antigen-independent human $\mathrm{T}$ cell adhesion. $J$ Immunol 1987;139:1037-45.

6 Sanchez-Madrid F, Krensky AM, Ware CF, et al. Three distinct antigens associated with human T-lymphocytemediated cytolysis:LFA-1, LFA-2 and LFA-3. Proc Natl mediated cytolysis:LFA-1, LFA-2

7 Tedder TF, Schmidt RE, Rudd CE, Kornacki MM, Ritz J Schlossman SF. Function of the LFA-1 and T4 molecules in the direct activation of resting human $B$ lymphocytes by T lymphocytes. Eur J Immunol 1986;16:1539-43.

8 Dustin ML, Springer TA. Lymphocyte function-associated antigen-1 (LFA-1) interaction with intercellular adhesion molecule-1 (ICAM-1) is one of at least three mechanisms for lymphocyte adhesion to cultured endothelial cells. $J$ Cell Biol 1988;107:321-31.

9 Dustin ML, Singer KH, Tuck DT, Springer TA. Adhesion of $\mathrm{T}$ lymphoblasts to epidermal keratinocytes is regulated by interferon $\gamma$ and is mediated by intercellular adhesion molecule 1 (ICAM-1). J Exp Med 1988;167:1323-40.

10 Griffiths CEM, Voorhees JJ, Nickoloff BJ. Characterisation of intercellular adhesion molecule-1 and HLA-DR of intercellular adhesion molecule-1 and HLA-DR recombinant gamma interferon and tumor necrosis factor. $J$ Am Acad Dermatol 1989;20:617-29.

11 Greve JM, Davis G, Meyer AM, et al. The major human rhinovirus receptor is ICAM-1. Cell 1989;56:839-47.

12 Vollger LW, Tuck DT, Springer TA, Haynes BF, Singe $\mathrm{KH}$. Thymocyte binding to human thymic epithelial cells is inhibited by monoclonal antibodies to CD-2 and LFA-3 antigens. J Immunol 1987;138:358-63.

13 Emilie D, Wallon C, Galanaud P, Fischer A. Olive D, Delfraissy J-F. Role of the LFA3-CD2 interaction in human specific B cell differentiation. J Immunol 1988, 141:1912-18.

14 Le P, Denning S, Springer T, Haynes B, Singer K. AntiLFA-3 monoclonal antibody induces interleukin 1 (IL1) release by thymic epithelial (TE) cells and monocytes. Fed Proc 1987;46:447. 
15 Stauder R, Greil R, Schulz TF, et al. Expression of leucocyte function-associated antigen-1 and 7F7-antigen, an adhesion molecule related to intercellular adhesion molecule-1 (ICAM-1) in non-Hodgkin lymphomas and leukaemias: possible influence on growth pattern and leukaemic behaviour. Clin Exp Immunol 1989:77:234-8.

16 Gregory $\mathrm{CD}$, Murray RJ, Ed Downregulation of cell adhesion molecules LFA-3 and phoma underlies tumor cell escape from virus-specific T phoma underlies tumor cell escape from virus-spe

17 Smith MEF, Marsh SGE, Bodmer JG, Gelsthorpe K, Bodmer WF. Loss of HLA-A,B,C allele products and lymphocyte function-associated antigen 3 in colorectal neoplasia. Proc Natl Acad Sci USA 1989;86:5557-61.

18 Van Noorden $S$. Tissue preparation and immunostaining techniques for light microscopy. In: Polak JM, Van Noorden S, eds. Immunocytochemistry. Modern methods and applications. Bristol: P G Wright 1986:26-53.

19 Ritter MA, Sauvage CA, Cotmore SF. The human thymus microenvironment: in vivo identification of thymic nurse microenvironment: in vivo identification of thymic nurse
cells and other antigenically distinct subpopulations of epithelial cells. Immunology 1981;44:439-46.

20 Law HY, Bodmer WF. Use of microimmobilisation and microagglutination assays for attempted detection of HLA antigens and $\beta 2 \mathrm{~m}$ on human sperm. Tissue Antigen 1978;12:249-69.

21 Denning SM, Dustin ML, Springer TA, Singer $\mathrm{KH}$ Haynes BF. Purified lymphocyte function-associated antigen-3 (LFA-3) activates human thymocytes via the CD2 pathway. J Immunol 1988;141:2980-5.

22 Mizutani S, Watt SM, Robertson D, et al. Cloning of human thymic subcapsular cortex epithelial cells with $\mathrm{T}$ lymphocyte binding sites and hemopoietic growth facto activity. Proc Natl Acad Sci USA 1987;84:4999-5003.

23 Adams DH, Hubscher SG, Shaw J, Rothlein R, Neuberge $\mathrm{JM}$. Intercellular adhesion molecule 1 on liver allograft during rejection. Lancet 1989;ii:1122-4.

24 Faull RJ, Russ GR. Tubular expression of intercellular adhesion molecule-1 during renal allograft rejection. Transplantation 1989;48:226-30.

25 Staunton DE, Dustin ML, Springer TA. Functional cloning of ICAM-2, a cell adhesion ligand for LFA-1 homologous to ICAM-1. Nature 1989;339:61-4. 